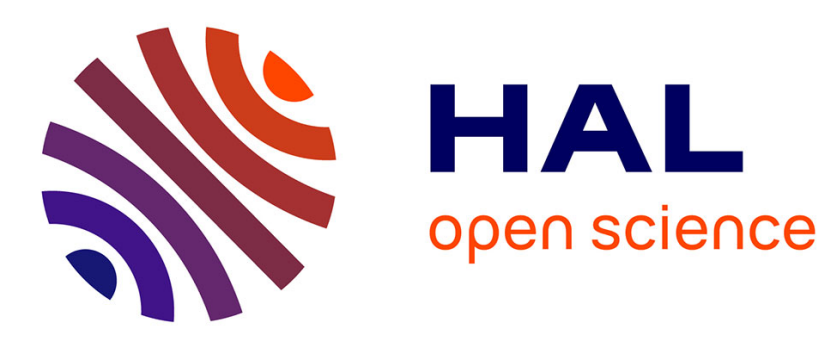

\title{
Low Speed Vehicle Localization using WiFi FingerPrinting
}

Dinh-Van Nguyen, Myriam Vaca Recalde, Fawzi Nashashibi

\section{To cite this version:}

Dinh-Van Nguyen, Myriam Vaca Recalde, Fawzi Nashashibi. Low Speed Vehicle Localization using WiFi FingerPrinting. International Conference on Control, Automation, Robotics and Vision, ICARCV 2016, Nov 2016, Phuket, Thailand. hal-01395973

\section{HAL Id: hal-01395973 \\ https://hal.inria.fr/hal-01395973}

Submitted on 13 Nov 2016

HAL is a multi-disciplinary open access archive for the deposit and dissemination of scientific research documents, whether they are published or not. The documents may come from teaching and research institutions in France or abroad, or from public or private research centers.
L'archive ouverte pluridisciplinaire HAL, est destinée au dépôt et à la diffusion de documents scientifiques de niveau recherche, publiés ou non, émanant des établissements d'enseignement et de recherche français ou étrangers, des laboratoires publics ou privés. 


\title{
Low Speed Vehicle Localization using WiFi FingerPrinting
}

\author{
Dinh-Van Nguyen, Myriam Elizabeth Vaca Recalde, Fawzi Nashashibi \\ RITS team - INRIA \\ Paris, France \\ dinh-van.nguyen@inria.fr, myriam.vaca-recalde@inria.fr, fawzi.nashashibi@inria.fr
}

\begin{abstract}
Recently, the problem of fully autonomous navigation of vehicle has gained major interest from research institutes and private companies. In general, these researches rely on GPS in fusion with other sensors to track vehicle in outdoor environment. However, as indoor environment such as car park is also an important scenario for vehicle navigation, the lack of GPS poses a serious problem. This study presents an approach to use WiFi Fingerprinting as a replacement for GPS information in order to allow seamlessly transition of localization architecture from outdoor to indoor environment. Often, movement speed of vehicle in indoor environment is low $(10-12 \mathrm{~km} / \mathrm{h})$ in comparison to outdoor scene but still surpasses human walking speed $(3-5 \mathrm{~km} / \mathrm{h}$, which is usually maximum movement speed for effective WiFi localization). This paper proposes an ensemble classification method together with a motion model in order to deal with the above issue. Experiments show that proposed method is capable of imitating GPS behavior on vehicle tracking.
\end{abstract}

Keywords-WiFi fingerprinting; low speed vehicle; indoor localization; autonomous vehicle; ensemble classification

\section{INTRODUCTION}

Indoor environment navigation is an important scenario for autonomous vehicle. A report in [1] estimates that $95 \%$ of time cars are in parking lot. In addition, studies in [2], [3] suggests that the average searching time for a free slot in car park in Paris and Lyon is 20 minutes and can be as high as 40 minutes for some districts. This leads to around 70 million hours of searching each year for France and represents a 700 million euro loss. In addition, several issues are identified as: noise pollution, insecurity, air pollution and $10 \%$ to $60 \%$ of congestion. These negative effects also cost France 70 million euro per annum. Thus, by pushing toward a completely autonomous navigation for indoor environment (particularly car park), it is possible that billions of euro will not be wasted every year.

In the last decade, there are a many researches in indoor positioning system with wide ranges of sensory approaches. Most of these researches focus on tracking human or indoor robots. Researches using vision techniques can be found in [4][7] which utilize camera (standard camera, depth-sensing camera, Kinect, etc.) to track target movement. These approaches achieve significant accuracy $(\sim 40 \mathrm{~cm})$ but lack of coverage range as well as costly implementation. Radio Frequency based approach is another method which is being adopted widely. Studies in [8]-[10] use WiFi signal to determine location of user in known environment. Companies like Google,
TABLE I. SUMMARY OF INDOOR LOCALIZATION SySTEM [13]

\begin{tabular}{|c|c|c|c|c|c|c|}
\hline & & \multicolumn{5}{|c|}{ Paraneters } \\
\hline & & Accuracy[m] & Coverage $[\mathrm{m}]$ & Cost & Complexity & $\begin{array}{c}\text { Typical } \\
\text { Environment }\end{array}$ \\
\hline \multirow{6}{*}{ Technologies } & Vision & $10^{-3} \div 10^{-1}$ & $1-10$ & High & High & Indoor \\
\hline & Infrared & $10^{-2} \div 1$ & $1-5$ & Medium/High & Low & Indoor \\
\hline & Ultrasound & $10^{-2}$ & $2-10$ & Medium & Low & Indoor \\
\hline & $W i-F i$ & $1 \div 10$ & $20-50$ & Medium/Low & Low & Indoor/Outdoor \\
\hline & RFID & $10^{-1} \div 1$ & $1-10$ & Low & Low & Indoor \\
\hline & Bluetooth & $1 \div 10$ & $1-30$ & Low & Low & Indoor/Outdoor \\
\hline
\end{tabular}

Apple, Polestar, etc. are using the concept of iBeacon, a low energy Bluetooth (BLE) beacon, as a low cost, easy to implement indoor localization solution. However this approach has low sampling rate as well as accuracy is in range of 1-3m [11][12]. A summary of indoor localization system can be found in Table I below.

Majority of these studies do not specify movement speed of tracking target. It is partly because movement speed of indoor objects can be assumed to be within $3-5 \mathrm{~km} / \mathrm{h}$ range (which is normal walking speed). However, this is an important factor when it comes to vehicle localization. Study in [2] shows that average speed of cars in car park is around $10-12 \mathrm{~km} / \mathrm{h}$ which doubles human walking speed. Together with sampling rate of sensors, localization accuracy may change dramatically when it come to vehicle tracking.

This paper proposes a solution that uses WiFi localization to replicate GPS behavior for indoor envrionment. An ensemble classification method of neural network is implemented together with motion model to overcome moving speed constraint. This is a first step in a fusion system consists of multiple localization technologies that aimed to have centimeter accuracy for indoor vehicle localization.

Section II gives a brief introduction to WiFi fingerprinting localization and a proposed improvement for noise filtering in data collection. Section III presents ensemble neural network structure. A constant speed motion model is adopted in section IV in order to improve localization result. Experiment setup and results are explained in section V. Section VI concludes the paper with future work.

\section{WIFI FINGERPRINTING METHOD}

Wifi Fingerprinting is a WiFi positioning solution which relies on an assumption: each location in environment has a 
unique set of signal strengths from all reachable Access Points (AP). Hence, by mapping that unique set of signal strengths and its corresponding coordinate in local map, the location can be derived later with only WiFi signal scan. Due to the availability of WiFi infrastructure, this method is a cost-effective solution and widely adopted for indoor positioning system.

There are two phases of WiFi fingerprinting: training phase and prediction phase. A training phase should be done by recording $\mathrm{WiFi}$ signal strengths of multiple reference positions in environment and labeling them with an ID. Each ID is then mapped to corresponding coordinate in local map. Set of all reference positions is considered to be classes of classification problem and noted as $\mathbf{P}$. In prediction phase, tracking target will run in the same environment of training phase while continuously scanning and recording WiFi signal. Each scanned data will be a vector of signal strengths without label. Trained classification method such as Random Forest, Neural Network, etc. will take this vector as input and return the most likely reference position which matches signal vector.

However, there are certain limitations of WiFi fingerprinting localization method. The major two are noisy data and low sampling rate. WiFi signal appears to be unstable under influence of environment factors. Problem such as multi-path propagation for which receiver receives multiple signals of same source, each travels in different path is a well-known issue. In addition for a normal $2.4 \mathrm{Ghz} \mathrm{WiFi}$ receiver, it takes in average 0.9 second to complete a scan of signals. This low sampling rate is capable of responding to average human walking speed ( $1.4 \mathrm{~m} / \mathrm{s}$ or $3-5 \mathrm{~km} / \mathrm{h}$ ). However, as we target vehicles such as cars, wheel chairs, etc. with speed of $10-12 \mathrm{~km} / \mathrm{h}(2.7-3.3 \mathrm{~m} / \mathrm{s})$, this low sampling rate introduces much higher error in localization. Thus, it is necessary to introduce a noise filtering method as well as a movement model to deal with these problems.

In training phase, One sampling vector of a reference position is described as in (1) where $x_{i, j}$ is WiFi signal strength from jth WiFi APs recorded in ith scan, $\rho_{l}$ is a label which has corresponding coordinate at position of sampling and $n$ is fixed constant (for learning algorithm purpose) which should be greater total number of APs in learning environment.

$$
\left\{x_{i, 1}, x_{i, 2}, x_{i, 3}, \ldots, x_{i, n}, \rho_{l}\right\}
$$

Collected data will be normalized in range of $[-1,1)$ where in particular scan, detected AP signals (so called RSSI Received Signal Strength Indicator) will be normalized (2) in range $[0,1)$ with 0 as weakest possible signal strength and 1 as strongest possible signal strength. Other signals will be scored as undetected APs at $\rho_{l}$ and take value -1 .

$$
x_{i}=\left\{\begin{array}{r}
-1, A P_{i} \text { undetected } \\
1-\frac{(-1) \times R S S I}{100}, A P_{i} \text { detected }
\end{array}\right.
$$

During the training phase, a fingerprinting database is formed as in (3) where $m$ is number of sampling vectors in training phase, $n$ is number of total APs and $l$ is number of labels (or reference position in training phase).

$$
D=\left\{\begin{array}{c}
x_{1,1}, x_{1,2}, x_{1,3}, \ldots, x_{1, n}, \rho_{1} \\
x_{2,1}, x_{2,2}, x_{2,3}, \ldots, x_{2, n}, \rho_{1} \\
x_{3,1}, x_{3,2}, x_{3,3}, \ldots, x_{3, n}, \rho_{2} \\
\vdots \\
x_{m, 1}, x_{m, 2}, x_{m, 3}, \ldots, x_{m, n}, \rho_{l}
\end{array}\right\}
$$

In order to reduce noise in collected training set, a spatial normalization process is applied. For each training vector, a global spatial mean value is computed in (4). The spatial normalization relies on assumption that varying signal strength of a small subset APs does not affect variance of all recorded APs. Thus by taking difference of each recorded signal with mean value, local noise by small set of AP signals will be removed (5). A new $m \times(n+1)$ matrix $\bar{D}$ is formed by substitute $\widehat{x_{l}}$ for each $x_{i}$.

$$
\begin{gathered}
\overline{x_{l}}=\frac{1}{N} \sum_{i=1}^{N} x_{i} \\
\widehat{x}_{\iota}=x_{i}-\overline{x_{l}}
\end{gathered}
$$

\section{ENSEMBLE CLASSIFICATION METHOD}

Ensemble learning is a well-known method of combining multiple learning models to derive better results of prediction[14]. In WiFi fingerprinting, especially for vehicle moving speed, the signal strength collected from WiFi Access Point is highly noisy. Often, noisy data with high variance lead to overfitting problem of classification method. One solution for this problem is applying Bagging (Bootstrap Aggregating) ensemble method [15].

Consider a classification method with a pair $\left\{X_{i}, Y_{j}\right\}$ where $X_{i}$ is a vector of predictor variable and $Y_{j}$ denotes a response, $Y_{j} \in\{1,2, \ldots m\}$. The target function is $P(Y=j \mid X=x)$ for classification. A function estimator which results from set of training samples and a classification model is formed (6).

$$
g(\cdot)=h\left(\left(X_{1}, Y_{1}\right),\left(X_{2}, Y_{2}\right), \ldots,\left(X_{n}, Y_{m}\right)\right)
$$

Bagging algorithm consists following steps:

Step 1: Construct a bootstrap sample (7) by randomly sampling with replacement $n$ times from original data:

$$
\left(X_{1}^{\wedge}, Y_{1}^{\wedge}\right),\left(X_{2}^{\wedge}, Y_{2}^{\wedge}\right), \ldots,\left(X_{\mathrm{n}}^{\wedge}, Y_{\mathrm{m}}^{\wedge}\right)
$$

Step 2: Compute bootstrapped estimator $g^{\wedge}(\cdot)$ (8) by applying same classification model to newly formed bootstrap sample.

$$
g^{\wedge}(\cdot)=h\left(\left(X_{1}^{\wedge}, Y_{1}^{\wedge}\right),\left(X_{2}^{\wedge}, Y_{2}^{\wedge}\right), \ldots,\left(X_{\mathrm{n}}^{\wedge}, Y_{\mathrm{m}}^{\wedge}\right)\right)
$$


Step 3: Repeat two steps above for $\mathrm{K}$ times with $\mathrm{K}$ is large. The bagging estimator is (9).

$$
g_{\text {bagg }}^{\wedge}(\cdot)=\frac{1}{K}\left(\sum_{i=1}^{K} g^{\wedge i}(\cdot)\right)
$$

Theoretically, the bagging estimator is (10) as $\mathrm{K}$ goes to infinity:

$$
g_{\text {bagg }}^{\wedge}(\cdot)=\mathbf{E}^{\wedge}\left[g^{\wedge}(\cdot)\right]
$$

Thus, a finite large $\mathrm{K}$ in practice is expected to improve accuracy of Monte Carlo approximation.

In this study, a neural network is constructed and loosely tuned (weak learner). Bagging method is implemented as following:

- Step 1: Construct a Bootstrap sample by repeatedly random sampling with replacement a row from original training matrix. $m$ random rows will be sampled to form a new $m \times(n+1)$ training data matrix.

- Step 2: Repeat process above for $\mathrm{K}-1$ times. In total, $\mathrm{K}$ training data matrices are formed (includes original data).

- Step 3: Train K neural networks on K matrices.

- Step 4: All K models make independent prediction on an input vector $\mathbf{X}$.

- $\quad$ Step 5: Each model returns a vector $\mathbf{V}=\left\{\mathcal{P}\left(\rho=\rho_{\mathrm{i}}\right) \mid\right.$ $\left.\rho_{\mathrm{i}} \in \mathbf{P}\right\}$ where $\mathbf{P}$ is set of all reference positions in training phase and $\mathcal{P}(\rho)$ is posterior probability that the vehicle is in a particular reference position. Take average of $\mathrm{K}$ resulting vectors then select one that has maximum probability. If that value surpasses threshold $h$, takes this as a valid prediction of input $\mathbf{X}$.

Choosing threshold $h$ is critical since it has huge impact on performance of localization. The lower value of $h$, the more predictions are accepted but also expected error is increased. With too high threshold, very few predictions are accepted so localization performance may not be satisfied.

\section{MOTION MODEL}

Due low frequency of WiFi scanning, it takes in average 0.9 second for 1 scan and prediction to be completed. Hence, a valid predicted location will have delayed result. Consider $S_{t 1}$ as predicted location result from neural networks, actual location at time of receiving result will be $S_{t 2}$ with $t 2-t 1=0.9$ second. Since $\Delta t=0.9$ is small enough, a simple constant speed model (11) is adopted:

$$
S_{t 2}=\left[\begin{array}{ll}
V & S_{t 1}
\end{array}\right] \cdot\left[\begin{array}{c}
\Delta t \\
1
\end{array}\right]
$$

Where $S_{t}=(x, y)_{t}$ with $\mathrm{x}, \mathrm{y}$ is coordinate. $\mathrm{V}$ is constant speed of vehicle obtained at time $t$.
In addition, the motion model will also help filtering out prediction. Since vehicle is moving with much higher speed in compare to sampling rate, one typical error is jumping prediction where predicted locations are jumping between adjacency reference points. Given prediction and correction from above step, a scoring operator will be applied on all possible reference points (classes) of WiFi localization in order to minimize jumping by a utilizing Euclidean distance cost function.

Every valid predictions (as define in section above) will increase the corresponding reference point score. A valid prediction is accepted when its score goes above a threshold and will be called valid location. In between a valid location and its successor valid prediction there are certain number of invalid predictions. A distance traveled between these two is computed by sum of all distance for each pair of scan in between (12). The result will then be compared to Euclidean distance of 2 reference points (13) corresponding to the valid location and valid prediction above and the difference must be under a tolerance $\varepsilon$ (14).

$$
d_{\text {travel }}=\sum \Delta X_{i}
$$

$$
d_{\text {ref }}=X_{\text {valid_prediction }}-X_{\text {valid_location }}
$$

$$
\left|d_{\text {travel }}-d_{\text {ref }}\right| \leq \varepsilon
$$

If the condition is not satisfied, the second prediction will not be accepted and its score will be lowered.

\section{EXPERIMENT \& RESULT}

\section{A. Experiment Setup}

The experiment is carried out in campus area of INRIARocquencourt with a cybercar (Fig.1) from RITS team. The cybercar is an electrical vehicle equipped with multiple sensors and a computer. For this study, following sensors are used: IMU (Inertial Measurement Unit), RTK GPS (Real Time Kinematic GPS) and a standard 2.4Ghz WiFi USB dongle.

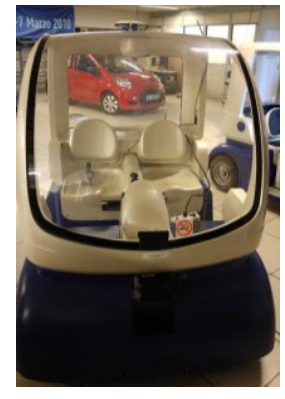

Figure 1. The cybercar 


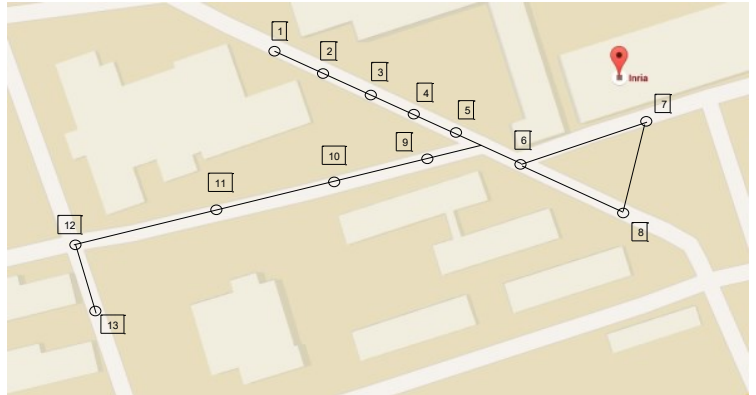

Figure 2. Experiment area with labeled position

The experiment is conducted in campus site, outdoor scene to make use of highly accurate RTK GPS for ground truth. 13 reference points are picked as illustrated in Fig. 2. A survey for average $\mathrm{WiFi}$ signal map and number of detected access points at each reference points are shown in Fig. 3, Fig. 4 respectively. Scale of WiFi signal strength will be in range $[0,1)$. Theoretically, highest possible WiFi signal strength will be $30 \mathrm{dbm}$ which equals to 0.7 in this scale.

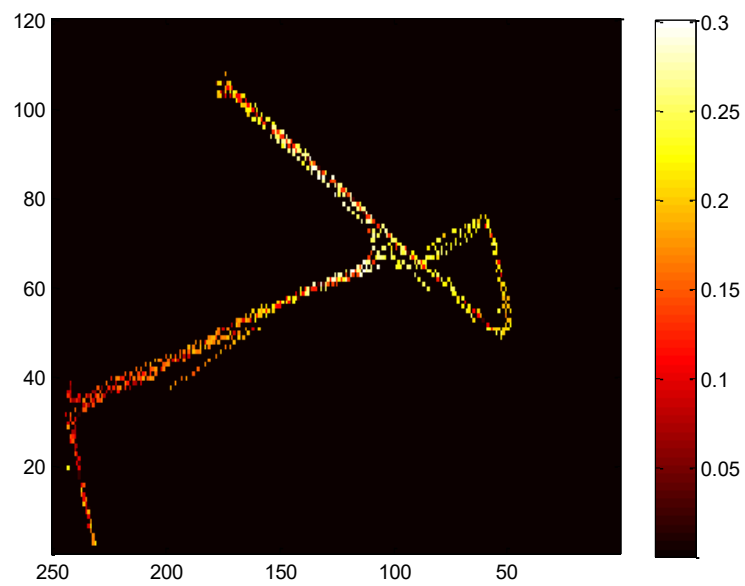

Figure 3. Average WiFi signal map

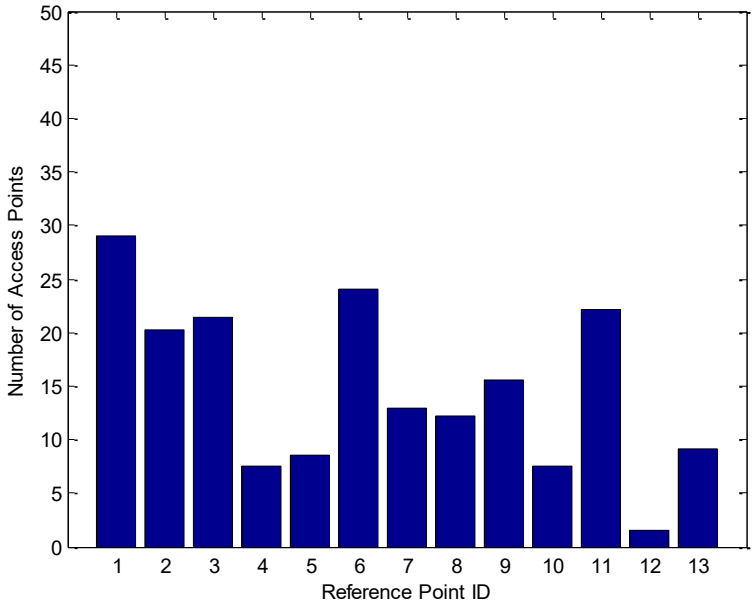

Figure 4. Number of Access Points detected in each reference location
Training phase consists of taking 30 sample data for each reference position. A database of 390 vectors are formed for 13 different classes. In prediction phase, the vehicle will make 3 round-trips starting from reference point 13, visit all reference points with maximum speed of $2.5 \mathrm{~m} / \mathrm{s}$. Localization results with threshold $h=0.65$ will be compared with RTK GPS result as ground truth. Details are shown in Table II. For $1264 \mathrm{~m}$ covered in the experiment, root mean square error is $6.85 \mathrm{~m}$ and maximum error is $16.48 \mathrm{~m}$. However, Fig. 5 shows an interesting observation that most of errors over $8 \mathrm{~m}$ are from reference points $11,12,13$.

TABLE II. LOCALIZATION RESULT

\begin{tabular}{|l|l|l|l|}
\hline Total Distance & Mean Error & RMS Error & $\begin{array}{c}\text { Maximum } \\
\text { Error }\end{array}$ \\
\hline $1264 \mathrm{~m}$ & $5.77 \mathrm{~m}$ & $6.85 \mathrm{~m}$ & $16.48 \mathrm{~m}$ \\
\hline
\end{tabular}

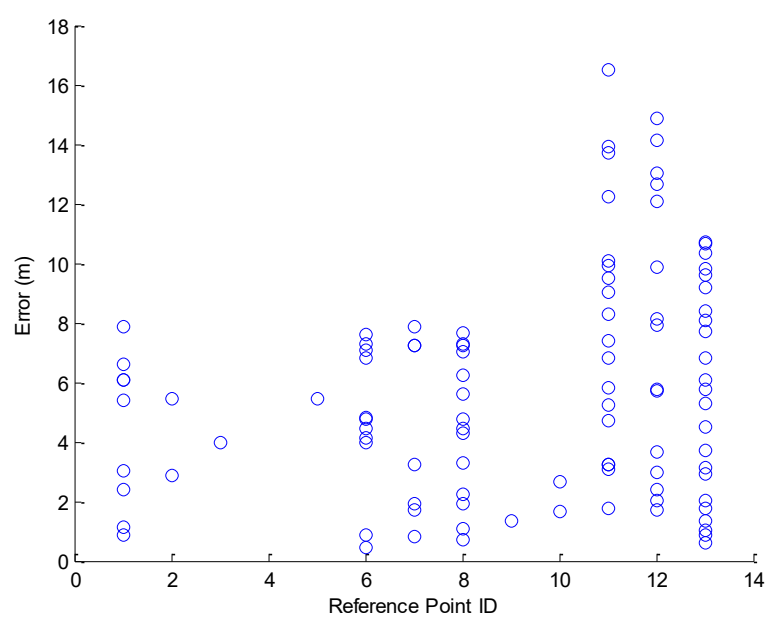

Figure 5. Distribution of error in each reference point

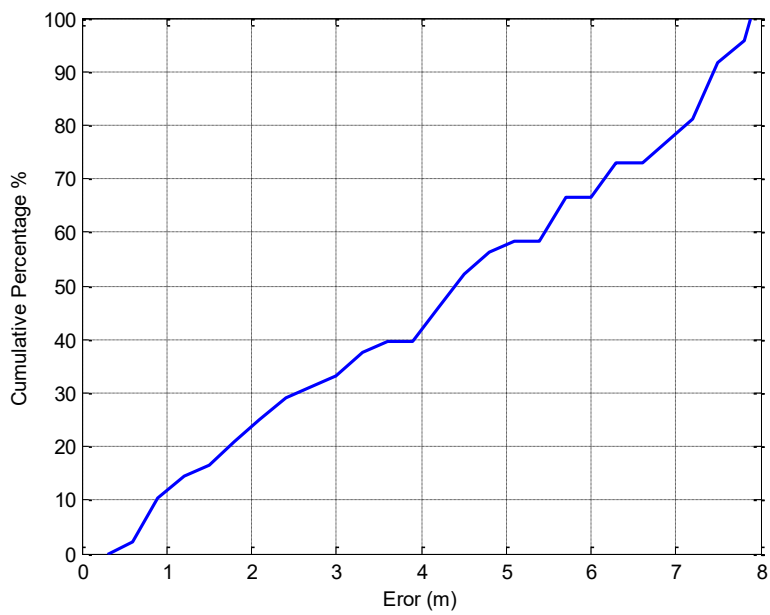

Figure 6. Error distribution for reference points $[1,10]$

These high errors are expected due to low average signal recorded at these locations demonstrated in Fig. 3 even though numbers of detected WiFi APs in reference points 11 and 13 are significant. Without these reference points, the maximum error 
TABLE III. CORRELATION BETWEEN SIGNAL STRENGTH AND LOCALIZATION ERROR

\begin{tabular}{|c|c|c|}
\hline $\begin{array}{c}\text { Average Signal } \\
\text { Strength Range } \\
\text { (dbm) }\end{array}$ & $\begin{array}{c}\text { Average Error } \\
\text { (m) }\end{array}$ & Maximum error (m) \\
\hline$[-100,-70]$ & 6.85 & 16.48 \\
\hline$[-80,-70]$ & 4.76 & 13.02 \\
\hline$[-75,-70]$ & 3.89 & 7.05 \\
\hline
\end{tabular}

is $7.88 \mathrm{~m}$ while root mean square error is $4.99 \mathrm{~m}$. In that case, error distribution is illustrated in Fig. 6. It shows that $90 \%$ of errors are achieved under $7.5 \mathrm{~m}$.

A further investigation shows in Table III indicates that there is a correlation between quality of signal strength to localization error. With better signal strength, localization performance appears to be enhanced significantly. Since experiment environment is an outdoor scene, it is worth to point out that quality of WiFi signal in this scene is much lower than indoor scene. Thus, a much better performance could be achieved in indoor environment.

\section{CONCLUSION AND FUTURE WORK}

This paper shows an early result of using WiFi localization for low speed vehicles to replicate GPS behavior. Although accuracy level is under expectation, it shows promising of using WiFi in combination with other technologies such as SLAM in order to have a seamless transition from outdoor localization system to indoor localization system.

The experiment result shows that with sufficient WiFi signal strength as well as number of visible APs, the result of localization can be improved significantly. In more than $1.2 \mathrm{~km}$ of testing environment with RTK GPS as ground truth, $8 \mathrm{~m}$ error is achieved for reference position with average signal strength in range of $[0.15,0.3]$ or $[-85 \mathrm{dbm},-70 \mathrm{dbm}]$. This result is expected to be improved in indoor condition with better WiFi signal.

In the future, environment map as well as a particle filter will be integrated into the solution to improve prediction result. Also, output from WiFi localization will be fused with other technologies in order to deliver reliable localization result.

\section{REFERENCES}

[1] P. Barter, “'Cars are parked 95\% of the time'. Let's check!,” 2013. [Online]. Available: http://www.reinventingparking.org/2013/02/carsare-parked-95-of-time-lets-check.html.

[2] S. Belloche, "On-street Parking Search Time Modelling and Validation with Survey-based Data," Transp. Res. Procedia, vol. 6, no. June 2014, pp. 313-324, 2015.

[3] E. Gantelet and A. Lefauconnier, "The time looking for a parking space: Strategies, associated nuisances and stakes of parking management in France," pp. 1-7, 2006.

[4] Q. Cai and J. K. Aggarwal, "Tracking Human Motion Using Multiple Cameras," Pattern Recognit., pp. 68-72, 1996.

[5] I. Bouchrika, J. N. Carter, and M. S. Nixon, "Towards automated visual surveillance using gait for identity recognition and tracking across multiple non-intersecting cameras," Multimed. Tools Appl., pp. 12011221, 2014.

[6] R. Clark, N. Trigoni, and A. Markham, "Robust Vision-based Indoor Localization," Proc. 14th Int. Conf. Inf. Process. Sens. Networks, pp. 378379, 2015.

[7] P. Mirowski, R. Palaniappan, T. K. Ho, B. Labs, M. Avenue, and M. Hill, "Depth Camera SLAM on a Low-cost WiFi Mapping Robot," vol. 1, no. 908, pp. 0-5.

[8] F. Lemic, A. Behboodi, V. Handziski, and A. Wolisz, "Increasing Interference Robustness of WiFi Fingerprinting by Leveraging Spectrum Information," Comput. Inf. Technol. Ubiquitous Comput. Commun. Dependable, Auton. Secur. Comput. Pervasive Intell. Comput. (CIT/IUCC/DASC/PICOM), 2015 IEEE Int. Conf., pp. 1200 - 1208, 2015.

[9] J. Biswas and M. Veloso, "WiFi localization and navigation for autonomous indoor mobile robots," Proc. - IEEE Int. Conf. Robot. Autom., pp. 4379-4384, 2010.

[10] J. Huang, D. Millman, M. Quigley, D. Stavens, S. Thrun, and A. Aggarwal, "Efficient, generalized indoor WiFi GraphSLAM," Proc. IEEE Int. Conf. Robot. Autom., pp. 1038-1043, 2011.

[11] "POLESTAR," 2016. [Online]. Available: http://www.polestar.eu/. [Accessed: 29-Apr-2016].

[12] “SenionLab," 2016. [Online]. Available: https://senionlab.com/. [Accessed: 29-Apr-2016].

[13] L. Mainetti, L. Patrono, and I. Sergi, "A survey on indoor positioning systems," 2014 22nd Int. Conf. Software, Telecommun. Comput. Networks, pp. 111-120, 2014.

[14] T. G. Dietterich, "Ensemble Methods in Mac hine Learning," Mult. Classif. Syst., vol. 1857, pp. 1-15, 2000.

[15] L. Breiman, "Bagging Predictors," Mach. Learn., vol. 24, pp. 123-140, 1996. 